\title{
Spontaneous Parity Violation
}

\author{
P.R. Crompton ${ }^{1}$ \\ 1 Institut für Theoretische Physik, Universität Leipzig, \\ Augustusplatz 10/11, D-04109 Leipzig, Germany *
}

(Dated: October 8, 2018)

\begin{abstract}
We disprove the Vafa-Witten theorem on the impossibility of spontaneously breaking parity in vector-like gauge field theories, identifying a mechanism driven by quantum fluctuations. With the introduction of a meromorphic Lattice formulation, defined over 5 dimensions, we demonstrate that the minima of the free energy can be distinct from the maxima of the partition function : identifying and evaluating a suitable contour for the partition function defined such that asymptotic behaviour of the complex action is non-oscillatory.

PACS numbers: 73.43.Nq, 11.15.Ha, 11.10.Gh.
\end{abstract}

Recently attempts have been made to determine the properties of the Grand Canonical Partition Function of QCD at nonzero baryon densities using numerical Lattice QCD approaches [1]. These results are directly relevant for determining the equation of state of QCD for experimental Heavy Ion Collider results. These efforts are frustrated numerically by the difficulties in constructing efficient sampling algorithms at finite baryon densities. One faces the problem of a complex-valued Lattice action, whose phase oscillates rapidly as a function of the configuration within a Lattice statistical ensemble. This then prevents the effective use of importance sampling methods. All recent sampling improvement treatments in this vein appear now to suffer from the same generic flaw of an unphysical light pseudo-baryonic transition [2, 3].

To gain understanding of this feature an analytic treatment of partition function zeroes for QCD and QCD-like theories with a complex action problem was recently proposed [4, 5]. Here the properties of the zeroes are identified as relating directly to specific regions of parameter space in the continuum where the partition function is itself ill-defined. This is linked in the discussion to the Vafa-Witten Theorem on the impossibility of spontaneously breaking parity symmetry in QCD [6]. A local bosonic parity breaking source term in the action is shown to generate a similarly pathologically ill-defined continuum partition function. This is demonstrated explicitly through the properties of the zeroes, and is used to justify the main theorem result. Although not included in this specific zeroes analysis the Vafa-Witten theorem result is understood to represent a wider pathology in Lattice simulation 7, 8]. Bosonic terms with substructure in the Vafa-Witten formalism give rise to unphysical diagrammatic contributions to the Lattice QCD partition function. For example, in $N_{f}=2 \mathrm{QCD}$ with a four fermion operator source [9], or the Aoki phase with Wilson fermions [10, 11, 12.

\footnotetext{
*Electronic address: crompton@itp.uni-leipzig.de
}

We now apply a new quantum system partition function zeroes treatment to this problem, and show that in contrast it is possible to give a well-defined continuum partition function for all generic complex action problems (of those of the form in the Vafa-Witten discussion) for all parameter space regions. Under the proviso, that, the local source term has a highly nonlocal component for which the continuum limit has been reached over an additional phase space dimension. We introduce a new quantum loop operator definition for the partition function and show that it is formally equivalent to the Vafa-Witten construction, but in addition, meromorphic. This new analysis is used to rigorously disprove the VafaWitten result, and demonstrate that it is in fact possible for any global Lattice symmetry (like taste) to be spontaneously broken via the system dynamics. Specifically, we demonstrate that the branch cut argument introduced in [4, 5, 13, 14] used to argue that the partition function is ill-defined is specious. The branch in fact can be resolved analytically and unambiguously for all Lattice system sizes, $N$, and in particular in the asymptotic limit.

The new analysis also indicates that unless the Lattice simulation of a quantum system with a complex action problem produces extremely finely balanced statistical averaging between degenerate vacua (namely that which in the asymptotic limit $N \rightarrow \infty$ connects smoothly with the continuum) then spurious phase behaviour will be observed. We have applied a new fine-balancing scheme recently, based on this understanding, via numerical simulation to several Lattice QCD models at finite baryon densities and independently quantum spin systems (which have a hidden continuous gauge symmetry) 3, 15, 16, 17, 18. This article then, in part, provides a generic proof of the existence of the continuum limit of these ongoing Lattice analyses, and the validity of our generic continuum matching program for these calculations.

The purpose of this new article is to establish the origin of large light disconnected contribution in recent lattice QCD schemes at finite baryon densities in a generic man- 
ner. Specifically, we wish to establish the relationship between the statistical ambiguities of numerical sampling and the parity symmetry violation arguments of Vafa and Witten. This is expressed through a saddle point point discussion of the partition function and is specific to our new quantum loop operator formulation. To do this, following Lee and Yang [19], we wish to now argue for separate treatment for the approach to the continuum limit of the zeroes polynomial in quantum systems, as opposed to classical systems. We therefore rederive the partition function zeroes polynomial proof of Lee and Yang and the validity of its approach to the continuum limit for the quantum case, formulated in terms of loop operators. In doing so, we demonstrate that this is the generic origin of these disconnected contributions in numerical simulations. This then has direct applicability in the design of new improved algorithms - to include correct quantum effects - both for Lattice QCD and quantum spin systems that are otherwise spoilt by multiplicative nonrenormalizable anomalies induced through the noncommutativity of finite Lattice system dynamics 20, 21].

This article is then organised as follows : in Section 凹 we introduce a new quantum loop operator formalism for the partition function and identify that this is formally equivalent to the local bosonic operator expansion on finite system size introduced in the Vafa-Witten discussion. We then consider the continuum limit of this formulation and demonstrate that the zeroes of the loop operator polynomial are formally equivalent to partition function zeroes, but only as defined with reference to a prior noncommuting continuum limit being taken over an additional dimension in an extended phase space. We then derive the saddle point solution for the partition function of this complex-valued quantum loop operator formulation.

In Section [II we discuss the analytic continuation of the saddle point solution with reference to the symmetry breaking properties of the newly defined quantum partition function, and the Vafa-Witten discussion. We demonstrate that the results can be analytically continued across the entire phase plane in the zeroes expansion parameter. Therefore any nonperturbative operator satisfying the quantum loop operator definition can be analytically continued throughout the complex phase-plane, and is an exact analytic solution to the complex action problem.

\section{QUANTUM LOOP OPERATORS}

The partition function, $\mathcal{Z}$, for a general continuous group $G$ we define in terms of quantum loop operators by,

$$
\mathcal{Z}_{N}(t)=\int \mathrm{d} g \exp \left[\int_{0}^{t} A_{g}\left(\boldsymbol{n}_{s}\right) z-V\left(\boldsymbol{n}_{s}\right) d s\right],
$$

with $t \in \mathbb{R}, z \in \mathbb{C}$,

$$
\begin{aligned}
A_{g}(\boldsymbol{n}) & \equiv \sum_{(i, j)}^{N \otimes T} \sum_{\sigma \in G} \lambda_{i j \sigma}(\boldsymbol{n}) \frac{\left\langle\boldsymbol{n} \oplus \mathbf{1}_{i \sigma} \oplus \mathbf{1}_{j \sigma} \mid g\right\rangle}{\langle\boldsymbol{n} \mid g\rangle} \\
V(\boldsymbol{n}) & \equiv \sum_{(i, j)}^{N \otimes T} \sum_{\sigma \in G} \lambda_{i j \sigma}^{\prime}(\boldsymbol{n}) \frac{\left\langle\boldsymbol{n} \oplus \mathbf{1}_{i \sigma} \oplus \mathbf{1}_{\sigma j} \mid \boldsymbol{n}\right\rangle}{\langle\boldsymbol{n} \mid \boldsymbol{n}\rangle}
\end{aligned}
$$

where $\lambda_{i j \sigma}(\boldsymbol{n}), \lambda_{i j \sigma}^{\prime}(\boldsymbol{n}) \in \mathbb{C},\{\sigma\}$ form some finite subset of the elements of $G$, and $g$ is a general element of $G$. We treat the complex-valued continuous symmetry generalisation of the problem considered in 22, 23]. The fact that the operator definition is diagonalisable implicitly defines a local gauge transformation on the Hilbert space,

$$
\begin{aligned}
& \sum_{(i, j)}^{N \otimes T} \sum_{\sigma=G} \lambda_{i j \sigma}(\boldsymbol{n}) \frac{\left\langle\boldsymbol{n}^{\prime} \oplus \mathbf{1}_{i \sigma} \oplus \mathbf{1}_{j \sigma} \mid \boldsymbol{n}\right\rangle}{\langle\boldsymbol{n} \mid g\rangle}\left\langle\boldsymbol{n}^{\prime} \mid g\right\rangle \\
& \quad=\left\langle\boldsymbol{n}^{\prime} \mid g\right\rangle\left\langle\boldsymbol{n}^{\prime}|A| \boldsymbol{n}\right\rangle\langle\boldsymbol{n} \mid g\rangle^{-1}=\left\langle\boldsymbol{n}^{\prime}\left|A_{g}\right| \boldsymbol{n}\right\rangle .
\end{aligned}
$$

We should compare this directly with the single plaquette model given in terms of the Vandermonde determinant discussed in 24, 25], which yields a similar form of time-ordered product. The connection to Vafa-Witten formalism is made explicit by noticing that equivalently the integration of the exponent in can be performed over the spatial Lattice volume via a change of basis, $\boldsymbol{n}_{s}^{\prime}$. The partition function is then given in the usual Vafa-Witten form,

$$
\begin{aligned}
\mathcal{Z}_{T}(z) & =\int \mathrm{d} g \exp \left[\int_{0}^{z} A_{g}\left(\boldsymbol{n}_{s^{\prime}}\right) t-V\left(\boldsymbol{n}_{s^{\prime}}\right) d s^{\prime}\right] \\
& =\int \mathrm{d} g \exp \left[\int_{0}^{z} A_{g}^{\prime}\left(\boldsymbol{n}_{s^{\prime}}\right) i t-V^{\prime}\left(\boldsymbol{n}_{s^{\prime}}\right) d s^{\prime}\right](5) \\
& A_{g}^{\prime}\left(\boldsymbol{n}_{s^{\prime}}\right)=\operatorname{Im}\left[A_{g}\left(\boldsymbol{n}_{s^{\prime}}\right)\right] \\
V^{\prime}\left(\boldsymbol{n}_{s^{\prime}}\right) & =V\left(\boldsymbol{n}_{s^{\prime}}\right)-\operatorname{Re}\left[A_{g}\left(\boldsymbol{n}_{s^{\prime}}\right)\right]
\end{aligned}
$$

where $V\left(\boldsymbol{n}_{s^{\prime}}\right) \in \mathbb{R}$ since it is hermitean in the previous basis, $\boldsymbol{n}_{s}$.

\section{A. Thermodynamic Limit Monotonicity}

It is surprisingly simple to prove the existence of the thermodynamic limit for partition function in (11). The proof consists of two parts. The first step is to identify the saddle point solution of the partition function. The second step is then to determine the limiting value of this asymptotic solution when the neighbourhood of the saddle point is extended to cover the entire phase space. Apart from isolated singularities the partition function is then necessarily holomorphic. This is the essence of Lee and Yang's discussion in [19, 26]. 
For the quantum case we face now an additional subtlety. This has not been previously addressed in the literature. Partition functions for quantum systems are defined over an extended phase space - a trace is taken over an additional dimension. Two examples are given above in (11) and (5). For a quantum system to be truly holomorphic, and the thermodynamic limit exist, it is therefore not merely sufficient that the thermodynamic limit be proven to exist over either $\boldsymbol{n}_{s}$ or $\boldsymbol{n}_{s^{\prime}}$. Rather, the thermodynamic limit must exist over $\boldsymbol{n}$. Expressing this another way, existing partition function studies invoking this holomorphic property over $\boldsymbol{n}_{s^{\prime}}$ (holomorphic in $z$ ) have a thermodynamic limit that is strictly speaking only defined up to infrared divergences (ie. they are not necessarily simultaneously holomorhpic in $t$ ). For Lee and Yang with classical systems, which have no trace relation, this complication does not arise. The partition function in this case is given directly over $\boldsymbol{n}$, without the time-ordering of the integrand.

We now prove the existence of the thermodynamic limit for the new quantum loop operator formulation for the partition function in (11). The saddle point, which maximises the partition function in some region of the extended phase space $M$, is defined through,

$$
R_{M} \equiv \sup _{M} R(\boldsymbol{m}),
$$

where $\boldsymbol{m}$ is parameterises some contour in $N \otimes T$, and $R$ is the integrand of the partition function. The value of the integrand in the neighbourhood of the saddle point, $M_{\delta}$, is defined through,

$$
R_{\delta}(\boldsymbol{m}) \equiv R(\boldsymbol{m})-R_{M} \leq 0 .
$$

Not every point contained in $M$ is necessarily in the neighbourhood of the saddle point. We consider how the neighbourhood evolves as the contour is increased in length : the limit where $\boldsymbol{m} \rightarrow \boldsymbol{n}$.

$$
\lim _{\boldsymbol{m} \rightarrow \boldsymbol{n}} R_{\delta}(\boldsymbol{m})=\lim _{\boldsymbol{m} \rightarrow \boldsymbol{n}} R(\boldsymbol{m})-R_{M} \leq 0 .
$$

The relation between the consecutive basis elements (along $\boldsymbol{m}$ ) is given partly through the local gauge symmetry transformation in (44). However, we can have a situation for some general group $G$ in which we have multiple degenerate solutions of 10. In this case a subspace must be projected out to time-order the integrand and resolve this degeneracy. With this resolution is then necessarily monotonic and decreasing, since the potential nonlocal ambiguities are removed. Next, taking the limit $\lim _{M \rightarrow N \otimes T}$ we have,

$$
\begin{aligned}
\lim _{M \rightarrow N \otimes T} & {\left[\lim _{\boldsymbol{m} \rightarrow \boldsymbol{n}} R_{\delta}(\boldsymbol{m})\right] } \\
= & \lim _{M \rightarrow N \otimes T}\left[\lim _{\boldsymbol{m} \rightarrow \boldsymbol{n}} R(\boldsymbol{m})-R_{M}\right] \leq 0 .
\end{aligned}
$$

This limit is also monotonic and decreasing with above choices, and therefore the thermodynamic limit of the quantum loop operator partition function in is welldefined. The holomorphic properties we are therefore interested in are those of the space $N \otimes T$, modulo, the time-ordering necessary to resolve the nonlocal ambiguities of the gauge group. It should be clear now from a numerical perspective that it is not necessary to perform either integral in the integrands exactly : in order to be simultaneously holomorphic in $t$ and $z$ it is sufficient for the integrand to cover $N \otimes T$.

\section{B. Saddle Point Determination}

We are interested to find an explicit expression for the evolution of the partition function, $\mathcal{Z}_{N}$, in the limit $N \rightarrow \infty, N>0$. There are exactly $N$ singularities in the partition function in as a consequence of the above monotonicity arguments. By construction, the loop operator definition of $\mathcal{Z}_{N}$ is meromorphic so we can equally well formulate the discussion in terms of the poles of $\mathcal{Z}_{N}$, $\left\{V_{k}\right\}$, or the zeroes, $\left\{\lambda_{k}\right\}$. The poles turn out to be easier to treat than the zeroes, but for clarity the relation between the poles and zeroes is given through,

$$
\begin{aligned}
A(z) & \equiv \prod_{k=0}^{N} \frac{z+\lambda_{k}}{z+V_{k}}, \\
\frac{1}{2 \pi i} \int_{\mathcal{C}} \frac{A^{\prime}(z)}{A(z)} d z & =\sum_{k} n\left(\mathcal{C}, \lambda_{k}\right)-\sum_{k} n^{\prime}\left(\mathcal{C}, V_{k}\right),
\end{aligned}
$$

where $n$ and $n^{\prime}$ are the respective homotopies of the zeroes and poles, and $\mathcal{C}$ is some contour homologous to 0 in $N \otimes T$. This property will become important shortly when we consider finite multiplicities for the singularities, or equivalently, global symmetries in $N \otimes T$.

The schema we now follow is the complex-valued generalisation of the problem considered in [27, 28]. Namely, we define the Laplace transform of the partition function, $\widetilde{\mathcal{Z}}_{N}$, as a function of the poles, $\left\{V_{k}\right\}$. Then we formulate the inverse Laplace transform that gives us back the partition function expressed as a function of $t, N$ and $\left\{V_{k}\right\}$. Finally, we evaluate this integral equation for $\mathcal{Z}_{N}$ via standard asymptotic series approaches in order to identify the dominant behaviour of the partition function in the above limit. In particular, this approach allows us to identify the saddle point solution of the partition function as distinct from the asymptotic convergence properties of the zeroes towards the $\operatorname{Re} z$ axis.

The Laplace transform of the partition function polynomial in at finite $N$ is given in terms of the poles of the partition function by,

$$
\widetilde{\mathcal{Z}}_{N}(z)=\int_{0}^{\infty} d t e^{-z t} \mathcal{Z}_{N}(t)=\epsilon^{N} \prod_{k=0}^{N} \frac{1}{z+V_{k}},
$$

where $z, \epsilon, V_{k} \in \mathbb{C}$. The inverse Laplace transform of is 
given by,

$$
\begin{gathered}
\mathcal{Z}_{N}(t)=\frac{1}{2 \pi i} \int_{\mathcal{C}} d z e^{z t} \widetilde{\mathcal{Z}}_{N}(z)=\frac{1}{2 \pi i \epsilon} \int_{\mathcal{C}} d z \exp [N \varphi(z)], \\
\varphi(z)=\frac{z t}{N}-\sum_{k=0}^{N} \frac{1}{N} \log \left(\frac{z+V_{k}}{\epsilon}\right) .
\end{gathered}
$$

The contour $\mathcal{C}$ in, is defined as any suitable path running between $\pm \mathrm{i} \infty$, for which any singular behaviour is appropriately treated. We want to now evaluate the asymptotic behaviour of the partition function separately for the two cases: when a parity symmetry is, and is not, realised on the vacuum.

In the first case we have no special symmetry relations defined between the poles, and $\varphi(z)$ is therefore of the generic form above in. Solving explicitly for $\varphi^{\prime}\left(z_{0}\right)=0$ we have,

$$
\sum_{k=0}^{N} \frac{1}{z_{0}+V_{k}}=t
$$

Note, the location of the saddle point, $z_{0}$, is a function of both $\left\{V_{k}\right\}$ and $t$. Potentially the saddle point lies to the left of some subset of the poles, $\left\{V_{m}\right\}, m \leq N$. Therefore the integral in is given by,

$$
\mathcal{Z}_{N}(t)=\frac{1}{2 \pi i \epsilon} \int_{\mathcal{C}_{z_{0}}} \exp [N \varphi(z)] d z+\sum_{l=1}^{m} \frac{e^{V_{l} t}}{\epsilon} \prod_{k \neq l}^{N} \frac{1}{V_{l}+V_{k}}
$$

where $\mathcal{C}_{z_{0}}$ is the contour that passes through $z_{0}$. For this first case, when one assumes no parity symmetry is realised with the gauge fields, the asymptotic behaviour of the partition function is therefore given by,

$$
\begin{aligned}
\lim _{N \rightarrow \infty, N>0} \mathcal{Z}_{N}(t) & =\frac{1}{2 \pi i \epsilon} \exp \left[N \varphi\left(z_{0}\right)-\frac{i}{2} \theta\left(z_{0}\right)\right]\left(\sqrt{\frac{-2 \pi}{N\left|\varphi^{\prime \prime}\left(z_{0}\right)\right|}}+O\left(N^{-3 / 2}\right)\right), \\
& =\exp \left[z_{0} t-\sum_{k=0}^{N} \log \left(\frac{z_{0}+V_{k}}{\epsilon}\right)-\frac{i}{2} \theta\left(z_{0}\right)\right]\left(\sqrt{\frac{1}{2 \pi \epsilon^{2} N\left|\varphi^{\prime \prime}\left(z_{0}\right)\right|}}+O\left(N^{-3 / 2}\right)\right),
\end{aligned}
$$

where $\theta\left(z_{0}\right)=\operatorname{Arg} \varphi^{\prime \prime}\left(z_{0}\right)$.

For the second case it is straightforward to define a parity symmetry action on $Z_{N}(t)$ simply by reversing the orientation of the elementary plaquettes in $N \otimes T$. This then yields the symmetry of the zeroes $\left\{\lambda_{k}, \lambda_{k}^{*}\right\}$. From the Argument Principle in (13) the poles are then given through,

$$
\begin{gathered}
\widetilde{\mathcal{Z}}_{N}(z)=\epsilon^{N} \prod_{k=0}^{N} \frac{1}{\sqrt{\left(z+V_{k}\right)\left(z+V_{k}^{*}\right)}}, \\
\varphi(z)=\frac{z t}{N}-\sum_{k=0}^{N} \frac{1}{N} \log \left(\frac{\sqrt{\left(z+V_{k}\right)\left(z+V_{k}^{*}\right)}}{\epsilon}\right) .
\end{gathered}
$$

The singularities at $z=\operatorname{Re} V_{k} \pm \operatorname{Im} V_{k}$ therefore correspond to branch points, with a branching exponent of $-1 / 2$. Again, we want to define the contour such that it passes through $z_{0}$, but this is again potentially obstructed by singularities lying to the right of $z_{0}$. We therefore choose the branch cut to correspond to this deformation over the saddle point. If $\operatorname{Im} V_{k}<0$ the deformation will start at the branch point and descend to $-i \infty$. If the $\operatorname{Im} V_{k}>0$ the deformation will start at the branch point and ascend to $i \infty$. If $\operatorname{Im} V_{k}=0$ the singularity is a pole, which we will treat as a residue contribution as before.
This choice of branch cut does not introduce an arbitrariness into the calculation. Although it is not now possible to analytically continue across the cut, $\varphi(z)$ is specified unambiguously along the original contour, and the analytic continuation between contours is also specified unambiguously. Crucially we keep the same branch cut for the pairs $\left\{V_{l}, V_{l}^{*}\right\}$. The cuts to the left and right of the contour deformation $\mathcal{C}$ are defined through,

$$
\left(z+V_{l}\right)_{+}^{-1 / 2}=e^{-\pi i}\left(z+V_{l}\right)_{-}^{-1 / 2} .
$$

The analogue of the residue contribution for the branch points is therefore,

$$
\begin{aligned}
\mathcal{Z}_{N}^{l}(t) & =\int_{\mathcal{C}} e^{N \varphi(z, k \neq l)}\left(z+V_{l}^{*}\right)^{-1 / 2}\left(z+V_{l}\right)_{-}^{-1 / 2} d z \\
& -\int_{\mathcal{C}} e^{N \varphi(z, k \neq l)}\left(z+V_{l}^{*}\right)^{-1 / 2}\left(z+V_{l}\right)_{+}^{-1 / 2} d z \\
& =2 \int_{\mathcal{C}} e^{N \varphi(z, k \neq l)}\left(z+V_{l}^{*}\right)^{-1 / 2}\left(z+V_{l}\right)_{-}^{-1 / 2} d z
\end{aligned}
$$




$$
\sim 2 e^{N \varphi\left(z=V_{l}, k \neq l\right)} \sum_{n=0}^{\infty} \frac{\Gamma(n+1 / 2) b^{n}(0)}{n ! N^{n+1 / 2}}
$$

where,

$$
\begin{gathered}
b(0)=\left(-2 i \operatorname{Im} V_{l} \cdot \varphi^{\prime}\left(V_{l}, k \neq l\right)\right)^{-1 / 2} \\
\varphi^{\prime}\left(z=V_{l}, k \neq l\right)=\frac{t}{N}-\sum_{k=0, k \neq l}^{N} \frac{1}{N} \frac{\operatorname{Re}\left(V_{l}+V_{k}\right)}{\sqrt{\left(V_{l}+V_{k}\right)\left(V_{l}+V_{k}^{*}\right)}} .
\end{gathered}
$$

and the $b^{n}(0)$ form the analogue of the Laurent series for the branch points. The solutions are not oscillatory in $N$, as was found with previous discussions. In our new formulation we can see as well that the behaviour of the branch points can wholly dominate the asymptotic form of the partition function in the case in which a parity symmetry is realised, solely dependent on the relative magnitude of the prefactor, $\exp \left[\varphi\left(V_{k}, t\right)\right]$.

We are able to reach this new point of unambiguous saddle point solutions for two reasons. Firstly, the new problem is defined over a higher dimension than previous discussions, so $z$ and $t$ are separated. This means that the singularities can be distinguished from asymptotic scaling. Secondly, because the new construction is meromorhpic we can identify all the singularities in $\mathcal{Z}_{N}$, which are of a finite number.

The above arguments also are readily generalised to cases with different branching exponents - corresponding to different global symmetries. Specifically, if the branching exponent is less than -1 then the above asymptotic identifications for the branches can be performed via integration by parts.

\section{SPONTANEOUS SYMMETRY BREAKING}

We have constructed in (11) a partition function that is formally equivalent to that of the Vafa-Witten discussion. It consists of a local bosonic path integral, on which is defined a symmetry breaking source term, $A_{g}^{\prime}\left(\boldsymbol{n}_{s^{\prime}}\right)$. To relate our saddle point identification further to the Vafa-Witten discussion on spontaneous parity violation we now need to evaluate the vacuum expectation of the source term. Since the path integral is time-ordered this is given by,

$$
\begin{aligned}
\langle\mathcal{O}\rangle & =\lim _{N \rightarrow \infty}\left(\frac{1}{N} \frac{\partial \log \left(\mathcal{Z}_{N}(t)\right)}{\partial t}\right) \\
& =\lim _{N \rightarrow \infty}\left(\frac{1}{N} \frac{\int d z z e^{z t} \widetilde{\mathcal{Z}}_{N}(z)}{\int d z e^{z t} \widetilde{\mathcal{Z}}_{N}(z)}\right) .
\end{aligned}
$$

We therefore have two possible solutions, from 222 and (26), for the case when parity is realised by the system,

$$
\langle\mathcal{O}\rangle=z_{0} \quad \text { or } \quad\langle\mathcal{O}\rangle=V_{l}
$$

In particular we can see for this second case in (26), associated with the branch point, that the free energy density minima does not correspond with the partition function maxima at $z_{0}$. What we can see through our new formalism is that at finite $N$ there is a finite subtraction to made to properly define the contour for the partition function, and this itself can give rise to a parity breaking contribution. From (22) at most one of these expectations can be zero simultaneously, and so the potential for spontaneous symmetry breaking is well-defined.

Our new analysis does not mean that it is particularly likely that parity be spontaneously broken in $\mathrm{QCD}$, since in our formulation it is identified as a quantum effect driven by the system dynamics through $\left\{V_{k}\right\}$. Plausibly, these symmetry breaking effects are only observed in instances where the statistical properties of the the Lattice ensemble become very much non quasi-classical, ie. the orders of the contributions in (22) and (26) become comparable [29, 30].

As a second result we should notice from that it is possible to analytically continue the partition function throughout the entire $z$ plane. By definition the partition function is holomorphic in this region. We can deduce in principle it is therefore possible to resolve the complex action problem via numerical simulation. If a lattice determination correctly determines the polynomial expansion in up to quantum fluctuations at any single point in $z$, ie. that generates a covering of the Hilbert space in (2) and (3), the system can be smoothly mapped to all point in the $z$ plane. Since the result smoothly maps to the continuum the Lattice evaluation is also sufficiently defined for the dynamics to generate a nonzero vacuum expectation value of the source, $\mathcal{O}$, and to observe spontaneous symmetry breaking in the continuum.

Conversely, we can understand the origin of the disconnected contributions in existing Lattice evaluations. Algorithms can be based around the principle that a global symmetry is exactly held, and equal Monte Carlo weights assigned for each branch. However, because only the positive definite contributions are kept, corresponding to real probabilistic weights, the opposite cut is in effect taken for each branch point multiplet in (21). Genuine quantum effects can never therefore be observed as the cancellation between sectors is exact configuration by configuration, by construction and furthermore the system is nonrenormalizable [31, 32].

\section{SUMMARY}

We have introduced a quantum loop operator formalism for the partition function of vector-like gauge field theories, and identified that this is formally equivalent to the local bosonic operator expansion on finite system size introduced in the Vafa-Witten discussion. The Lattice formulation is given over an extended phase space $N \otimes T$, and the source parameter is itself discretized in the new approach. We have then considered the contin- 
uum limit of this space and identified that the zeroes of the loop operator polynomial are formally equivalent to partition function zeroes. Although this result is distinct from classical systems requiring a prior noncommuting limit to be taken over $T$ to ensure the formulation is meromorphic. The saddle point solution for the asymptotic behaviour of this complex-valued partition function was then determined. Spontaneous symmetry breaking is well-defined for case in which a parity symmetry is re- alised by the system. In the latter case the free energy minima can be distinct from the partition function maxima, and the generalisation of the result to general global symmetries was identified.

By construction, the formulation can be analytically continued across the entire phase plane in the zeroes expansion parameter. Therefore, any nonperturbative operator satisfying the quantum loop operator definition is an exact solution to the complex action problem.
[1] E. Laermann and O. Philipsen, Ann. Rev. Nucl. Part. Sci. 53 (2003) 163.

[2] K. Splittorff, [hep-lat/0505001].

[3] P.R. Crompton, Nucl. Phys. Proc. Suppl. 119 (2003) 550.

[4] V. Azcoiti, V. Laliena and A. Galante, in Guangzhou 2000, Nonperturbative methods and lattice $Q C D$, edited by X.Q. Luo and E.B. Gregory (Singapore, World Scientific, 2001). [hep-lat/0007045].

[5] V. Azcoiti and A. Galante, Phys. Rev. Lett. 83 (1999) 1518.

[6] C. Vafa and E. Witten, Phys. Rev. Lett. 53 (1984) 535.

[7] T. Cohen, Phys. Rev. D 64 (2001) 047704.

[8] T. Cohen, in From fields to strings, Vol. 1, edited by M. Shifman, [hep-ph/0405043].

[9] S. Sharpe and R. Singleton Jr., Phys. Rev. D58 (1998) 4501.

[10] S. Aoki, Phys. Rev. D 30 (1984) 2653.

[11] S. Aoki, Phys. Rev. Lett. 57 (1986) 3136.

[12] S. Aoki and A. Grotsch, Phys. Rev. D45 (1982) 3845.

[13] X.D. Ji, Phys. Lett. B 554 (2003) 33.

[14] M. Aguado and M. Asorey, [hep-th/0204130].

[15] P.R. Crompton, Nucl. Phys. B 619 (2001) 499.

[16] P.R. Crompton, Nucl. Phys. B 626 (2002) 228.

[17] P.R. Crompton, in Strong and Electroweak Matter 2002: Proceedings, edited by M.G. Schmidt (River Edge, N.J.,
World Scientific, 2003). [hep-lat/0301001].

[18] P.R. Crompton, W. Janke, Z.X. Xu and H.P. Ying, Nucl. Phys. Proc. Suppl. 140 (2005) 817, [hep-lat/0408041].

[19] T.D. Lee and C.N. Yang, Phys. Rev. 87 (1952) 404.

[20] G. Cognola and S. Zerbini, Nucl. Phys. B602 (2001) 383.

[21] P. R. Crompton, [hep-lat/0504014].

[22] M. Ostilli and C. Presilla, J. Phys. A 38 (2005) 405.

[23] M. Beccaria, C. Presilla, G. de Angelis and G. JonaLasinio, Europhys. Lett. 48 (1999) 243.

[24] V. Periwal, [hep-th/9908203].

[25] A. Dumitru, R.D. Pisarski and D. Zschiesche, [hep-ph/0505256].

[26] M. Aizenman, B. Nachtergaele, Commun. Math. Phys. 164 (1994) 17.

[27] M. Ostilli and C. Presilla, JSTAT 0504 (2005) 007.

[28] R. Balian, G. Parisi, and A. Voros, Phys. Rev. Lett. 41 (1978) 1627.

[29] D. E. Khareez, R. D. Pisarski and M. H. G. Tytgat, Phys. Rev. Lett. 81 (1998) 512.

[30] D. E. Khareez and R. D. Pisarski, Phys. Rev. D61 (2000) 1901.

[31] M. Creutz, Phys. Rev. Lett. 92 (2004) 201601.

[32] H. Neuberger, Phys. Rev. D70 (2004) 097504. 